\title{
Polypyrrole-gold nanostructured composite, active and durable electrocatalytic material
}

\author{
Sylwia Malinowska • Marianna Gniadek - Tomasz Rapecki • \\ Eliza Kurek • Zbigniew Stojek • Mikołaj Donten
}

Received: 30 December 2013 / Revised: 19 January 2014 / Accepted: 21 January 2014 / Published online: 16 February 2014

(C) The Author(s) 2014. This article is published with open access at Springerlink.com

\begin{abstract}
The consequences of treatment of gold nanocrystals present in polypyrrole-gold composites and electrodeposited gold nanocrystals (PPY-Au and Au NPs) with OH radicals generated in Fenton's reaction were investigated. Particularly, the changes in the morphology and the electrochemical properties of those materials are shown. For both materials, the etching effect was noticed. Contrary to significantly reduced catalytic activity, the changes observed in size and shape of gold nanostructures were less pronounced. In the case of PPY-Au composite material, the etching effects were less intense. Even after a 60-min radical treatment of the PPYAu composite material, the gold-nanocrystal catalytic activity remained high. The limited dissolution of the gold nanocrystals in the PPY-Au composites, compared to bare $\mathrm{Au}$ NPs, can be explained by the presence of the polymer which served as a kind of protective barrier against the oxidizing agent. A decrease in the electrocatalytic properties vs. the electrooxidation of ethanol of both forms of gold nanocrystals were observed after the treatment with hydroxyl radicals.
\end{abstract}

Keywords Gold nanoparticles · Polypyrrole $\cdot$ OH radicals · Composite $\cdot$ Electrocatalytic properties

\section{Introduction}

Composite materials containing conducting polymers and noble metal nanoparticles have been used as electrode- and electrodemodifying materials [1-4], contact catalysts [5], sensor- [6] and biosensor substrates [7-9], and in several other applications [10]. These types of materials have unique electrochemical

S. Malinowska · M. Gniadek · T. Rapecki • E. Kurek · Z. Stojek ·

M. Donten $(\bowtie)$

Faculty of Chemistry, University of Warsaw, Pasteura 1,

02-093 Warsaw, Poland

e-mail: donten@chem.uw.edu.pl properties, and the search for the methods leading to either an enhancement or a decrease in their electrocatalytic properties attracts chemists.

It is known that the hydroxyl radicals generated in Fenton's reagent quickly dissolve gold asperities or small irregularities from mechanically polished gold surfaces which make the surfaces much smoother [11, 12]. Such treatment of the regular gold-electrode surface caused the reduction of its electrocatalytic properties. The surface of a gold nanocrystal does not have mechanically formed asperities; however, its surface is usually very well developed. So, a good point was how $\mathrm{OH}$ radicals can alternate Au NPs. It has already been found that separated gold nanoparticles treated with Fenton's reagent lose substantially their electrocatalytic activities. Interestingly, the size and the total surface area were reported to change negligibly during the hydroxyl-radical treatment [13].

We have decided to examine the effect of Fenton's reagent on the structure and electrocatalytic properties of polypyrrolegold nanostructured composite materials and find out whether the polymer environment can protect the nanocrystals. The results were compared with those obtained for separated $\mathrm{Au}$ nanocrystallites. To obtain thin layers of the PPY-Au composite material on the glassy carbon electrode surface, the dip-in procedure was used. The principles of this easy and effective technique were described in reference [14]. The used procedure of formation of noble metal-conducting polymer composite is based on the electroless redox liquid-liquid interphase synthesis $[15,16]$.

\section{Experimental}

Chemical reagents

Thirty percent chloroauric acid, $30 \%$ hydrogen peroxide, acetic acid, and ethylenediaminetetraacetic acid (EDTA) were 
purchased from POCH S.A (Poland). Pyrrole and sodium perchlorate monohydrate were purchased from Fluka. Nitrobenzene, ammonium iron(II) sulfate hexahydrate, sodium acetate trihydrate, ethanol, sodium hydroxide, and potassium dicyanoaurate(I) were purchased from Aldrich. All reagents were of p.a. purity. Water was obtained from a Milli-Q Plus/Millipore purification system (conductivity of water: $0.056 \mu \mathrm{S} \mathrm{cm}^{-1}$ ).

\section{Instrumentation}

The SEM images were taken with a FE-SEM Merlin (Zeiss, Germany) instrument controlled by producer's software. The device was working at a low sample current to give the highest possible resolution and detailed structure imagining. Under such conditions, the effect of charging-up was diminished. The chamber pressure was $1.10^{-5}$ Torr, and pictures were registered for 3- and $15-\mathrm{kV}$ electron beam energy to obtain the best quality images. Before exposing the samples to vacuum, they were washed with ethanol and water and dried.

Voltammetric measurements were performed in the three-electrode system using a VersaSTAT 4 (Ametek, USA) potentiostat. A saturated calomel electrode (SCE) was used as the reference electrode, and a platinum wire served as the auxiliary electrode. A graphite rod electrode of $0.5 \mathrm{~mm}$ in diameter and a glassy carbon disk (GC, $3 \mathrm{~mm}$ in diameter; BASi) served as the working electrodes.

The ICP-MS measurements were done using an ICP-MS PerkinElmer, model Elan 9000, instrument. The samples for analysis were acidified with nitric $(\mathrm{V})$ acid up to $5 \%$ concentration.

\section{Synthesis of PPY-Au composite}

PPY-Au composites tightly covering surfaces of the solid substrates were obtained using the dip-in method; the synthesis occurred at the boundary of two immiscible liquids [14]. The glassy carbon disk electrodes were first carefully washed with water and then dried. Next, they were immersed for $2 \mathrm{~min}$ in a $1 \mathrm{M}$ solution of pyrrole (monomer) in nitrobenzene diluted with acetone in a 1:2 ratio. After that, the electrodes were removed from the monomer solution, and the dissolved acetone was evaporated in air for $5 \mathrm{~s}$ to obtain a thin film of monomer solution in nitrobenzene on the electrode/substrate surface. Afterward, the NB-monomer covered objects were immersed in a $0.1 \mathrm{M} \mathrm{AuCl}_{4}^{-}$(oxidant) aqueous solution for $15 \mathrm{~min}$. Then, the substrate samples were immersed in acetone and ethanol to remove nitrobenzene and in water to remove remains of the oxidant. Finally, they were dried in air.
Electrodeposition of Au NPs

Pure gold nanoparticles were electrodeposited on the GC electrode surface from $10 \mathrm{mM} \mathrm{KAu}(\mathrm{CN})_{2}$ solution in $0.1 \mathrm{M}$ $\mathrm{NaClO}_{4}$. Before the electroreduction process, the GC surface was polished with 1 - and $0.3-\mu \mathrm{m}$ alumina powders and rinsed with water. Then, a constant potential $(-1.65 \mathrm{~V})$ was applied. The electrode was polarized until the charge of reduction of the gold(I) complex reached $5 \mathrm{mC}$. This charge was equivalent to the amount of gold embedded in the PPY matrix estimated as $10 \mu \mathrm{g}$.

Procedure for treating PPY-Au composite and Au NPs with Fenton reagent

The GC electrodes covered with a thin layer of PPY-Au composite were immersed in a solution of acetate buffer $(10 \mathrm{mM}, \mathrm{pH} 4.7)$ containing also $1 \mathrm{mM}$ ethylenediaminetetraacetic acid and $1 \mathrm{mM}$ solution of ammonium and ferrous sulfates. Appropriate volume of a fresh $\mathrm{H}_{2} \mathrm{O}_{2}$ solution was added to the buffer just before the treatment; the desired concentration of $\mathrm{H}_{2} \mathrm{O}_{2}$ was $10 \mathrm{mM}$. It is known from the literature [11] that the time of action of the hydroxyl radical is very short, and therefore the graphite substrate was exposed to the Fenton reagent for $5 \mathrm{~min}$. This procedure was repeated several times with freshly prepared solutions of Fenton reagent. The overall exposure time was in the range from 5 to $60 \mathrm{~min}$. After the treatment, the electrodes were washed with water and dried.

\section{Results and discussion}

To examine the changes in electrochemical activity of the polypyrrole-Au (PPY-Au) composite materials caused by the $\mathrm{OH}$ radicals, we decided to use GC electrodes and cover them with thin layers of that composite. The composite layers attached to the carbon electrodes were obtained by the dip-inmethod [14]. The quality and the reproducibility of formation of the layers in this process were satisfactory. The conditions applied during the electroless liquid-liquid interface deposition gave layers of average thickness of PPY film ca. $0.5 \mu \mathrm{m}$ containing $10 \mu \mathrm{g}$ of nanostructured $\mathrm{Au}$. We expected that the conducting polymer present in the composite material might deactivate radicals and therefore act as a protective agent that would neutralize the etching effects of an aggressive medium. The results obtained with the PPY-Au nanostructural composites were compared with those for the gold nanocrystals electrodeposited directly on the GC surface. Figure 1a and c presents typical SEM images obtained before hydroxylradical treatment of the composite material and bare gold nanocrystals, respectively. In both cases, on the surface of the GC electrodes, the same amount of nanostructured gold 
$(10 \mu \mathrm{g})$ was placed by electroless deposition (Fig. 1a) and by electroplating (Fig. 1c), respectively. In the PPY-Au composite material, the gold nanoobjects (rosettes) were made up of nanosized rods and ultrathin flakes contrary to pure gold deposit that had a more compact form. The gold rosettes that were found in the composite were uniformly distributed and not bigger than $1 \mu \mathrm{m}$. The gold nanocrystals placed directly on the carbon electrode surface (in the absence of polymer matrix) obtained using the constant potential deposition technique did not exhibit the rosette-like structure. They got more regular forms resembling ovals, triangles, and squares (see Fig. 1c). The average size of those crystals was in the range of 200 to $400 \mathrm{~nm}$. They often formed flat groups that usually consisted of up to several units. They were also uniformly distributed on the surface of GC electrode.

The effects of the hydroxyl-radical etching of gold immobilized on the electrode surface (deposited in polymer or on plain carbon) were monitored for 60 min while exposed to Fenton's solution. Since the radical solutions are not enough stable [11], each 1-h treatment of the sample was divided into 5-min periods. For every 5-min treatment, a new portion of Fenton's reagent was prepared, and all solutions were marked and collected for further analyses.

In the first step of the examination of $\mathrm{OH}$-radical influence on the gold nanostructures that are in contact with PPY, SEM images of the composite film were taken. A comparison of the micrographs shown in Fig. 1a and c let us conclude that delicate damages were done to the Au surface during 12.5min-long etching periods (total time of exposition: $60 \mathrm{~min}$.). Similarly, small differences caused by the $\mathrm{OH}$-radical etching were observed for the bare gold nanocrystals electrodeposited on the GC electrode. It was apparent that the solid-metal cores of the nanostructures survived $1 \mathrm{~h}$ treatment, and only the outer details of the gold objects became blunt (more rounded). In general, the sharp edges and dendrite-like forms disappeared (see Fig. 1d). The rosettes placed in polymer matrix lost their spherical shape and became somewhat flatter (see Fig. 1b), which proves that $\mathrm{OH}$ radicals more effectively dissolve the exposed to the solution gold placed above the polymer film. The treatment with $\mathrm{OH}$ radicals did not lead a significant loss in the nanocrystal mass. The results for bare $\mathrm{Au}$ nanocrystals are in agreement with those of Dutta and Yang [13]; they reported that there were no serious differences between the structure of bare Au NPs before and after treating with the hydroxyl radicals.

Contrary to the above observations, the dissolution of bare noble metal electrodes took place in the presence of $\mathrm{OH}$ radicals. Nowicka et al. [11] reported on the dissolution of the mechanically formed asperities on the Au electrode surface treated with Fenton's reagent. They showed that hydroxyl radicals smoothed effectively the Au surface which was accompanied by the dissolution of significant amount of metal. Mirkin et al. [17] observed the dissolution of the platinum electrode surface during electrocatalytic oxygen reduction reaction at moderately negative potentials in organic medium. During that reaction, the radicals were generated and could damage the nano- and micro-Pt electrodes.

More changes in the structure and surface composition of the PPY-Au materials caused by radical attack could be noticed by using a SEM Backscattered Electron detector working in the low energy loss mode. The contrast visible on the images reflects the surface composition of the material. Figure $2 \mathrm{a}$ and $\mathrm{b}$ presents the composition of the PPY-Au layer before and after short treatment of it with the hydroxyl
Fig. 1 SEM images of untreated PPY-Au (a) and Au nanocrystals without polymer (c) and treated for 60 min with Fenton's reagent: PPY-Au (b), Au nanocrystals without polymer (d)
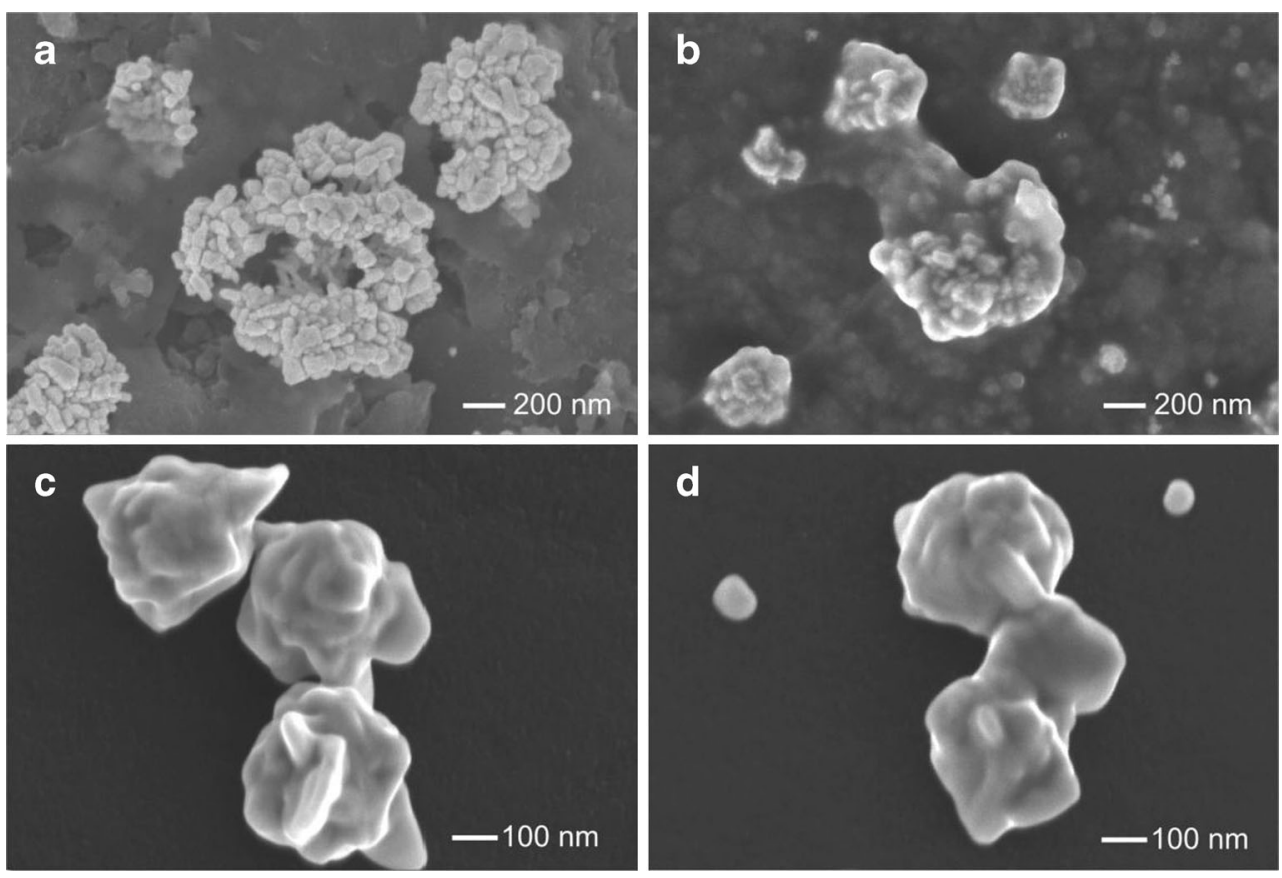
radicals. The bright objects correspond to the places where the gold structures are present, and the dark area is related to the polypyrrole matrix. As it is seen in Fig. 2a, before the treatment of the PPY-Au composite with Fenton's reagent, the brightness of gold rosettes is depressed. The micrographs of shortly (5 min) treated composites, taken under the same conditions, show much brighter gold surface. It suggests that in newly synthesized composite the gold nanostructures are covered with a very thin (circa few nanometers) layer of polypyrrole. After exposing the rosettes to a short-time radical treatment, the removal of the ultrathin polymeric film covering the rosettes occurred (see Fig. 2b). The formation of these ultrathin polymer layers on the surface of the gold rosettes was possible because there was an excess of the oxidant in the solution during the polymerization process. Probably, the layer was formed slowly at the end of the polymerization process using the remaining small amount of the monomer that escaped to the aqueous solution.

To confirm the fact, that a part of the gold nanoparticles was oxidized and dissolved by the hydroxyl radicals, the cumulative content of this metal in consecutive Fenton's solutions was summed up. The contents of the Au ions in the consecutive solutions obtained after every 5 -min treatment (up to total $60 \mathrm{~min}$ ) of the same sample were determined by the ICP-MS technique. Figure 3 shows those results obtained for the composite and the pure gold deposit.
The plots shown in Fig. 3 indicate that during the first $15 \mathrm{~min}$, the dissolution of gold nanoparticles embodied in PPY is more intensive compared to bare Au nanocrystals, just because the surface of these nanocrystals is much more developed, and the dissolved parts jutted over the polymer. After that time, the rate of Au dissolution $(\mathrm{dm} / \mathrm{dt})$ is smaller for the composite. Apparently for longer treatment times, the loss of gold would be bigger in the case of bare Au nanocrystals. The results of the ICP-MS measurements confirmed the previous SEM observations that the hydroxyl radicals cause, in a small degree, the etching of the gold nanocrystallites and in particularly small degree the etching of the composites.

\section{Change of catalytic properties}

The most important step in the investigation was the evaluation of the loss of the catalytic properties due to the treatment of the Au nanocrystals with the hydroxyl radicals. Similarly as it was done above, the PPY-Au composite was compared with the bare gold nanocrystals. Both materials were deposited on GC disk electrodes. For the examination of electrocatalytic activity of Au nanocrystals, the electrooxidation process of ethanol in alkaline aqueous solution was selected.

Figure 4a presents background cyclic voltammograms (GC electrode covered with PPY-Au composite material) obtained in $0.1 \mathrm{M}$ solution of $\mathrm{NaOH}$ before $(0 \mathrm{~min})$ and after $(60 \mathrm{~min})$
Fig. 2 Low energy loss BSE SEM images of PPY-Au composites before (a) and after (b) treatment with Fenton's reagent. SEM SE images of PPY-Au composites before (c) after $60 \mathrm{~min}(\mathbf{d})$ of treatment with Fenton's reagent
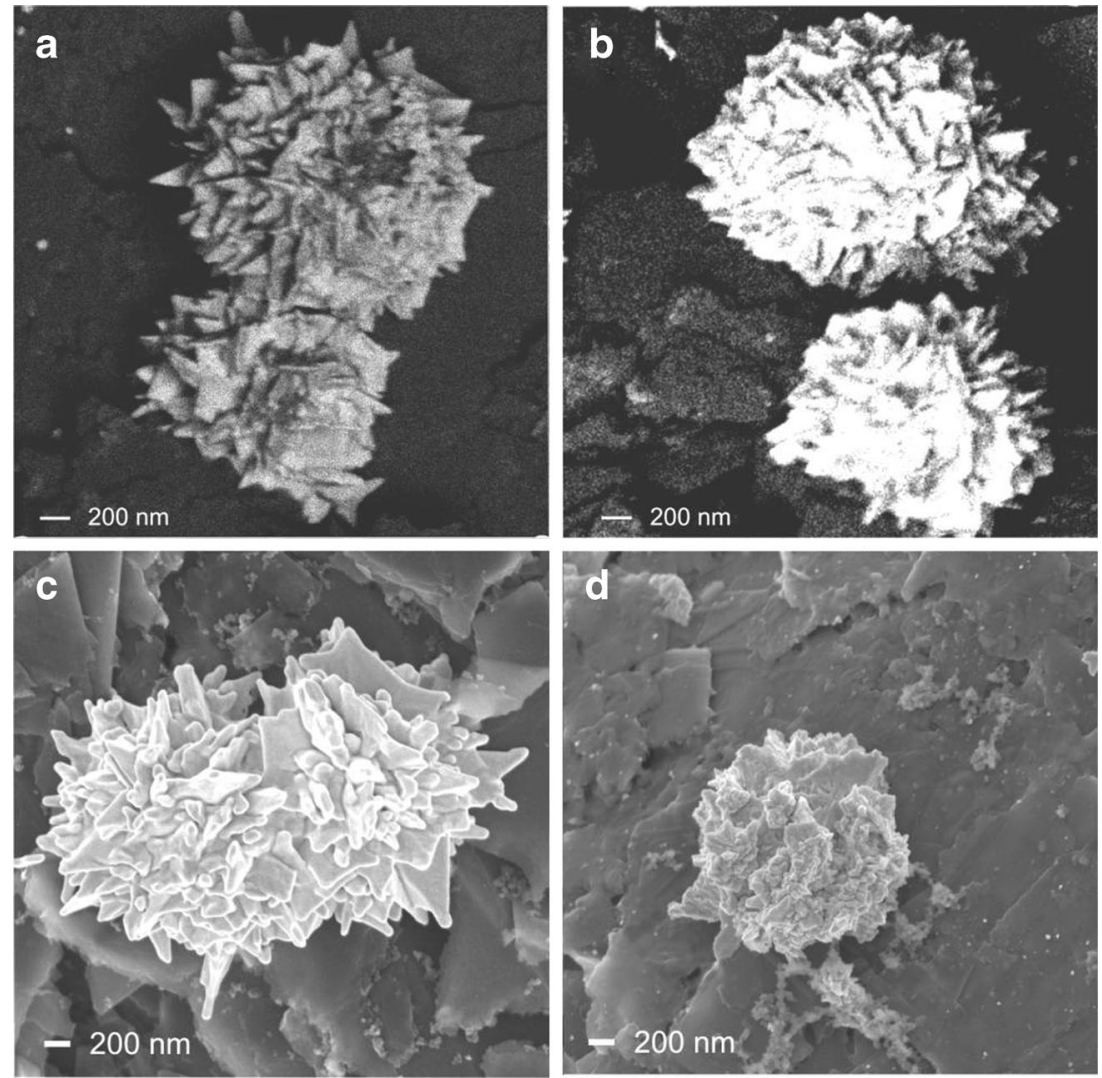


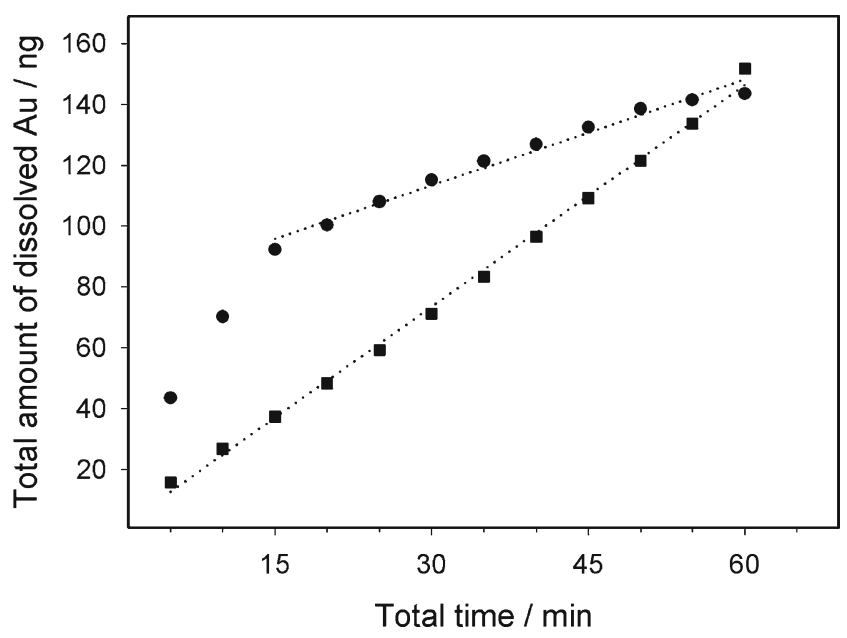

Fig. 3 Cumulated Au content in Fenton's solutions determined by ICPMS after consecutive treatments of PPY-Au (circles) and bare Au nanocrystals (squares) with $\mathrm{OH}$ radicals. Total treatment time is indicated in horizontal axis

the hydroxyl-radical treatment. A very broad peak in the potential range of $\mathrm{Au}$ oxidation was seen before treating the electrode with the hydroxyl radicals. After the treatment, this oxidation peak was much smaller and better defined probably due to removal of the thin PPY layer from the surface of the gold rosettes. The oxidation current decreased from $10 \mu \mathrm{A}$ to circa $7 \mu \mathrm{A}$. For GC electrodes covered by bare Au NPs, only small changes in anodic and cathodic peaks were observed (see Fig. 4b). In the voltammograms, the peak of electroreduction of oxygen at circa $-0.3 \mathrm{~V}$ is also seen

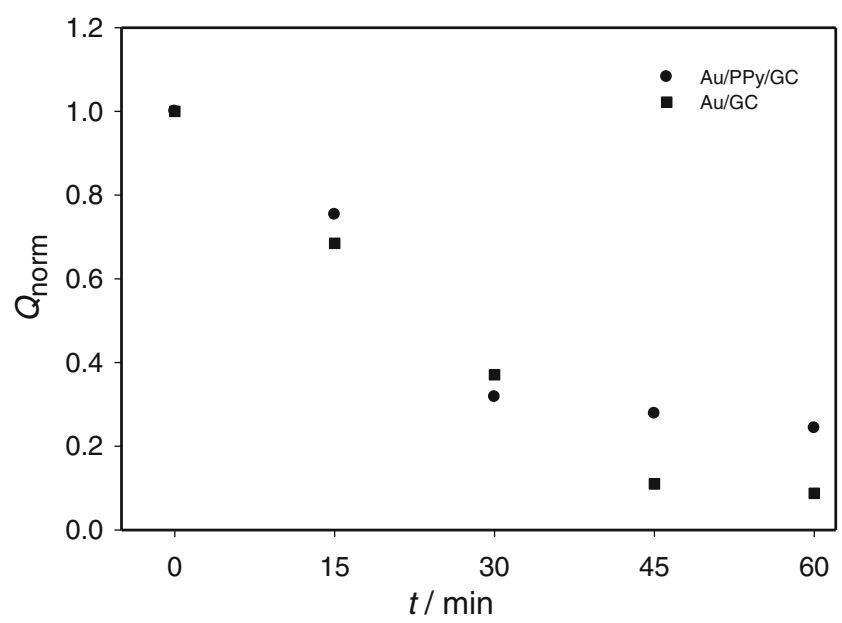

Fig. 5 Normalized charges of oxidation of ethanol in function of time of treatment of PPY-Au NPs (circles) and bare Au NPs (squares) with hydroxyl radicals

(solution was not degassed). That peak was practically not affected by the radical treatment.

The catalytic electrooxidation of ethanol at the PPY-Au composite and the bare gold nanoparticles deposited on GC substrate is shown in Fig. $4 \mathrm{c}$ and d. Before the treatment of the PPY-Au nanocomposite material with Fenton's reagent, the anodic electrooxidation current was very high. As a result of the exposure to the hydroxyl radicals, a strong decrease in the oxidation peak height was observed. After a 60 -min treatment, the measured currents dropped from 380 to $80 \mu \mathrm{A}$. In the case of bare $\mathrm{Au}$ NPs, not treated with $\mathrm{OH}$ radicals, the catalytic
Fig. 4 Cyclic voltamperograms for gold-containing polypyrrole $(\mathbf{a}, \mathbf{c})$ and pure gold NPs $(\mathbf{b}, \mathbf{d})$ both deposited onto glassy carbon disk electrode. Solution: $0.1 \mathrm{M}$ $\mathrm{NaOH}(\mathbf{a}, \mathbf{b})$ and $0.5 \mathrm{M}$ ethanol in $0.1 \mathrm{M} \mathrm{NaOH}(\mathbf{c}, \mathbf{d})$. Time of treatment of electrodes with $\mathrm{OH}$ radicals is indicated in graphs
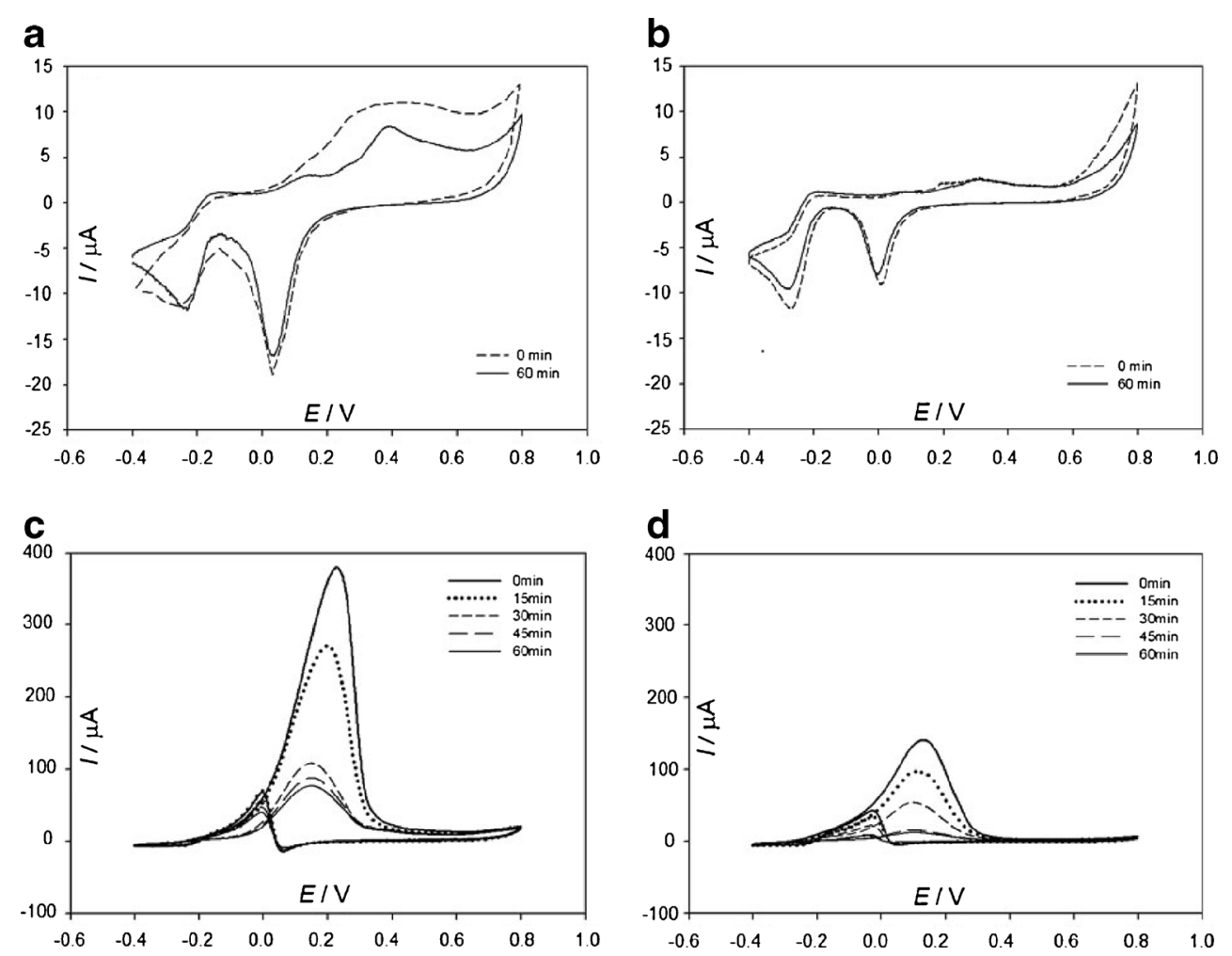
current equaled $140 \mu \mathrm{A}$, ca. 2.7 times lower than that obtained for the not-treated PPY-Au layer. Moreover, the decrease in the oxidation peak height (at bare Au NPs compared to composite) caused by the radicals attack was relatively more rapid. After each subsequent 15 -min radical treatment, the anodic current decreased by ca. $45 \mu \mathrm{A}$. After a 60 -min hydroxyl-radical treatment of the gold nanocrystals, the oxidation peak of ethanol reached the value of $10 \mu \mathrm{A}$ and was hardly visible (see Fig. 4d). These results revealed that the electrocatalytic activity of the electrodeposited gold nanoparticles decreased more rapidly than the electrocatalytic activity of the gold nanocrystals embedded in the polymeric network. Apparently, due to the presence of the polypyrrole in the composite, the resistance of the PPY-Au material to the hydroxyl-radical attack was higher than the resistance of pure gold crystals. In particular, after longer exposure times, the specific active planes in the PPY embedded nanocrystals were better protected.

Figure 5 presents the results of analysis of the charge of catalytic oxidation of alcohol in function of time of treatment of modified substrates with the hydroxyl radicals. The higher actual values of the anodic charge were observed in the case of PPY-Au layers. Also, the relative drop in the oxidation charge in time was smaller in the case of PPY-Au NPs, which indicated, particularly for longer treatment times, the more stable catalytic activity of the examined PPY-Au NPs material compared to bare Au NPs.

\section{Conclusions}

The performed experiments proved that gold nanoparticles are more resistant to $\mathrm{OH}$-radical attack compared to common bare gold polycrystalline electrodes. The total mass loss, in a 60min period, for bare gold NPs and embodied into PPY was circa twice lower than that reported by Nowicka et al. for mechanically polished polycrystalline gold [11]. For that comparison, we have recalculated the corresponding Au losses corresponding to identical geometric areas of the electrodes. Interestingly, the dynamics of gold dissolution, within the analyzed 60-min period and for examined materials, differed substantially. For polycrystalline gold, the dissolution rate decreased noticeably with time, while for Au nanocrystallites, it was rather constant in time. In fact, for the nanocomposites in the initial period of dissolution (the first 15-min), it was much faster and finally was the slowest among the examined materials. We relate the initial fast dissolution of Au in PPY to more developed and partially uncovered surface of gold nanocrystals. The final slowest dissolution of gold nanocrystals embedded in PPY was apparently a result of polymer protection.

Another interesting finding was that the treatment of the $\mathrm{Au}$ nanocrystallites with $\mathrm{OH}$ radicals had practically no influence on the oxygen reduction reaction while the alcohol oxidation reaction was affected strongly. Apparently, the crystal planes with highest electrocatalytic activity towards alcohol oxidation were also affected most by the action of radicals. The extraordinary property of nanocrystals towards oxidation of bigger molecules is generated by the presence of odd crystallographic structures on the nanocrystal surface, particularly, the atom orientations typical for steps between terraces. This phenomenon was well discussed and illustrated for methanol oxidation reaction (MOR) at platinum nanocrystals by S.W. Lee et al. [18].

Acknowledgments Support for this work by a Polish NCN Grant No. 2011/01/B/ST4/03032 and a Grant No. IP 2011045071 from the Polish Ministry of Science and Higher Education is gratefully acknowledged. The SEM images were obtained using the equipment purchased within CePt Project No.: POIG.02.02.00-14-024/08-00. S.M. thanks for a Mazovia PhD fellowship.

Open Access This article is distributed under the terms of the Creative Commons Attribution License which permits any use, distribution, and reproduction in any medium, provided the original author(s) and the source are credited.

\section{References}

1. Patil SD, Pawar SA, Devan RS, Gang MG, Ma YR, Kim JH, Patil PS (2013) Electrochemical supercapacitor electrode material based on polyacrylic acid/polypyrrole/silver composite. Electrochim Acta 105: 569-577

2. Rapecki T, Donten M, Stojek Z (2010) Electrodeposition of polypyrrole-Au nanoparticles composite from one solution containing gold salt and monomer. Electrochem Commun 12:624-627

3. Pandey RK, Lakshminarayanan V (2012) Ethanol electrocatalysis on gold and conducting polymer nanocomposites: a study of the kinetic parameters. Appl Catal B Environ 125:271-281

4. Hepel M (1998) The electrocatalytic oxidation of methanol at finely dispersed platinum nanoparticles in polypyrrole films. J Electrochem Soc 145:124-134

5. Xue K, Xu Y, Song W (2012) One-step synthesis of 3D dendritic gold@polypyrrole nanocomposites via a simple selfassembly method and their electrocatalysis for $\mathrm{H}_{2} \mathrm{O}_{2}$. Electrochim Acta 60:71-77

6. Li J, Xie H, Li Y (2012) Fabrication of gold nanoparticles/ polypyrrole composite-modified electrode for sensitive hydroxylamine sensor design. J Solid State Electrochem 16:795-802

7. Valentini F, Galache Fernàndez L, Tamburri E, Palleschi G (2013) Single walled carbon nanotubes/polypyrrole-GOx composite films to modify gold microelectrodes for glucose biosensors: study of the extended linearity. Biosens Bioelectron 43:75-78

8. Donten M, Stojek Z (2012) In: Hepel M, Zhong C-J (eds) Nanoparticles and nanostructured materials used in modification of electrode surfaces. ACS, Washington, DC

9. Hepel M (2012) In: Hepel M, Zhong C-J (eds) Functional gold nanoparticles for biointerfaces. ACS, Washington, DC

10. Li J, Han J, Xu T, Guo Ch BX, Zhang H, Wang L, Sun H, Yang B (2013) Coating urchin-like gold nanoparticles with polypyrrole thin shells to produce photothermal agents with high stability and photothermal transduction efficiency. Langmuir 29:7102-7110 
11. Nowicka AM, Hasse U, Hermes M, Scholz F (2010) Hydroxyl radicals attack metallic gold. Angew Chem Int Ed 49:1061-1063

12. Nowicka AM, Hasse U, Sievers G, Donten M, Stojek Z, Fletcher S, Scholz F (2010) Selective knockout of gold active sites. Angew Chem Int Ed 49:3006-3009

13. Dutta G, Yang H (2011) Effect of Fenton's reagent on the electrocatalytic activity of gold nanoparticles. Electrochem Commun 13:1328-1331

14. Gniadek M, Modzelewska S, Donten M, Stojek Z (2010) Modification of electrode surfaces: deposition of thin layers of polypyrrole-Au nanoparticle materials using a combination of interphase synthesis and dip-in method. Anal Chem 82:469-472

15. Gniadek M, Bak E, Stojek Z, Donten M (2010) Metal ion-driven synthesis of polyaniline composite doped with metallic nanocrystals at the boundary of two immiscible liquids. J Solid State Electrochem 14:1303-1310

16. Gniadek M, Donten M, Stojek Z (2010) Electroless formation of conductive polymer-metal nanostructured composites at boundary of two immiscible solvents. Morphology and properties. Electrochim Acta 55:7737-7744

17. Noël J-M, Yu Y, Mirkin MV (2013) Dissolution of Pt at moderately negative potentials during oxygen reduction in water and organic media. Langmuir 29:1346-1350

18. Lee SW, Chen S, Suntivich J, Sasaki K, Adzic RR, ShaoHorn Y (2010) Role of surface steps of Pt nanoparticles on the electrochemical activity for oxygen reduction. J Phys Chem Lett 1: $1316-1320$ 